\title{
An Efficient Energy Utilization based Cluster Head Selection for Mobile Sensor Network
}

\author{
Shilpa Singh Rajput \\ Computer Science and Engineering Department UIT, \\ BU \\ Bhopal, India
}

\author{
Divakar Singh \\ Computer Science and Engineering Department UIT, \\ BU \\ Bhopal, India
}

\begin{abstract}
The sensor nodes in WSN (Wireless Sensor Network) are energy dependent and each and every functioning is only possible to exhaust some amount of power or energy. The WSN is decentralized network and nodes are free to move in limited radio range for communication. The routing protocol is performing important role for communication and better routing strategy is reduces the energy consumption and enhances the utilization of energy. LEACH is the energy efficient protocol and this protocol is only designed for energy based routing. In this paper we proposed a new multipath routing strategy with higher energy node selection scheme for selection of Cluster Head $(\mathrm{CH})$ and route establishment. The performance of proposed LEACH protocol is compare with normal LEACH protocol in WSN. The energy utilization of nodes is improves if the possibility of link breakage is reduced and the proposed energy approach is established strong link in dynamic network. The proposed scheme ignores the shortest path routing procedure but established the reliable path in between source to destination. The packet retransmission is consumes the lot of extra energy for sending or forwarding the same data and also not sure, in next time data is successful deliver to destination. The multipath routing is improves the reliability of link in terms of mobility. If the node/s is the part of link and goes to out of range then instantly the alternative route is present for data delivery in dynamic network. The network performance is measure through performance metrics like throughput, routing overhead and number of survive nodes in dynamic network.
\end{abstract}

\section{Keywords}

LEACH, Proposed LEACH, Multipath, Routing, Energy, WSN.

\section{INTRODUCTION}

A Wireless Sensor Network is (WSNs) [1] consisting of large number of battery-powered low-cost sensing devices, called sensor nodes. The main task of a sensor node is to sense an event in its vicinity and in response to the event generate \& transmits data to a remote sink. Sensors interface with a small computing device called node in WSN and perform communication using short range wireless transmitters. Such nodes form logical network autonomously but cooperatively in which data packets are routed hop-by-hop towards management nodes, called base stations or sinks. A wireless sensor network comprises a potentially large set of nodes that may be distributed over a large geographical area, outdoor or indoor. WSN enable countless sensing and monitoring services in vital importance area such as efficient industry production, safety and security at home, and in environmental monitoring and traffic. Patterns of Traffic in sensor networks can be derived from the physical processes that they sense. Sensor network typically operate under light load and suddenly become active in response to a detected, monitored
event.Sensors are normally battery-powered and left unattended after deployment. Also in most of the cases it is not possible to recharge or replace batteries. Thus efficient use of energy becomes a challenge for prolonging network lifetime in WSN.WSN are wireless networks consisting of spatially distributed autonomous devices using sensors to cooperatively monitor environmental or physical conditions, such as temperature, sound, vibration, pressure, motion, and pollutants, among others, at different locations. Applications using sensors are being increased. A wide range of them is now deployed in areas of civilian like object tracking ,habitat observation health monitoring etc.

\section{ROUTING PROTOCOLS}

In WSN dynamic network the topology are frequently changes that are the cause of link breakage. The direct connection in between sender and receiver is rarely possible. The connections are created as multip-hop till the destination is not found. The routing protocol is play a important role at network layer for data accepting and forwarding through each router or node. The data is sending by sender and accepted by receiver in thst procedure routing strategy is very important part of communication. For connecting to destination and data delivery the routing protocol is necessary for routing the data in between sender to receiver. Every routing protocol has different routing strategy of connection establishment but has same method of select shortest path in between sender and receiver. The shortest path is decided on the basis of minimum hop count value in MANET [2, 3]. The classifications of routing protocols in MANET are as follows:-

\subsection{Proactive Routing Protocol}

The proactive routing protocols are also known as table driven routing protocol and these routing protocols are maintaining the routing information of each node that are participating in routing procedure. In Mobile Ad hoc network the topology in network is changes by that the overhead of maintain the information of each and every node is very difficult and required huge amount of memory for storing routing information in network. In ad hoc network if the nodes are moves at slow speed then that protocol is supposed to be better for communication. The DSDV routing protocol is the example of proactive routing protocol.

\subsection{Reactive Routing Protocol}

The Reactive routing protocols are also known as on demand routing protocol and these routing protocols are maintaining the routing information on the basis of requirement of request receives by the neighbour. There is no information regarding routing is stored of each node that are participating in routing procedure. In Mobile Ad hoc network the topology in network is changes by that the overhead of maintain the information of each and every node is not needed to maintained. In ad hoc 
network if the nodes are moves at random speed then that protocol is supposes to be better for communication. The AODV routing protocol is example of reactive routing protocol.

\subsection{Hybrid Routing Protocol}

Since proactive and reactive both protocols work best in oppositely different scenarios, hybrid method uses both the protocols. It is mainly used to find a good balance between both protocols. Operations of proactive are restricted to small domain, whereas, reactive protocols are useful for locating nodes outside those domains.

\section{LITERATURE SURVEY}

The Aarti jain , B.V.R.Reddy in [1] have discussed EEC SCH . They compared EEC-SCH with LEACH-ERE, LEACH and WCA.

They had proposed a new method of energy efficient cluster head selection method. This was accomplished by electing sink as one of the cluster head for one of the clustering round.

Advantage of this is the neighbour nodes of sink has been relieved from the duties of being cluster head.

Area of improvement is issue of energy holes created near sink node.

M. Tripathi,, M.S. Gaur, V. Laxmi and R.B. Battula in [4] discuss an Energy Efficient LEACH-C (EELEACH-C) protocol, in which base station runs a sorting algorithm to obtain a list of candidate cluster head nodes sorted in descending value their residual energy. After examining the candidate cluster head nodes it selects those with maximum residual energy and then calculate the quadratic sum of the distances from each cluster heads to its member nodes to find the optimal solution.

Advantage Experimental result attests that the proposed protocol improves network longevity.

Simulation results show that the EELEACH-C has extended the lifetime of the network by $10 \%$ in comparison of LEACH and $5 \%$ from the LEACH-C.

Jingqing Wang and Xi Zhang in [5] describe the AQDBPSK/ DS-CDMA (alternating quadrature differential binary phase shift keying/direct-sequence code division multiple access) based energy-efficient and interference-mitigating scheme for the $3 \mathrm{D}$ clustered WCSNs with the minimum target-object coverage rate constraint. First, they apply the new low energy adaptive clustering hierarchy (NEW LEACH) architecture into our 3D WCSNs for interference mitigation and energy efficiency . Then, using the Nakagami- $m$ model, they develop the novel DS-CDMA based AQ-DBPSK modulation scheme to implement the interference-mitigating and energy efficient wireless communications over our 3D clustered WCSNs. Author also derive the optimal data rate to optimize the transmit-power and data-rate trade-off. Finally, they conduct the simulations evaluations, showing that their proposed schemes outperform the other existing schemes.

Jitender Grover, Reena Rani in [6] define the Wireless Sensor Network (WSN) popularity has increased tremendously in recent times. It made up of small nodes with sensing, computation, and wireless communication capabilities. It needs an efficient approach that makes judicious use of energy constrained wireless sensor networks, thus Probabilistic Density Based Adaptive Clustering (PDBAC) scheme is proposed to improve the survivability of network using LEACH (Low Energy Adaptive Clustering Hierarchy) protocol. The results of MATLAB simulation show that PDBAC scheme is more energy efficient in terms of dead nodes, throughput and balance energy of nodes. Average efficiency is also greater than Basic LEACH and I-LEACH, thereby extending the survivability of network.

In terms of routing overhead PDBAC scheme is inefficient but overall total efficiency is still more than LEACH and ILEACH.

Advantage of this work is simplicity

Vinay Kehar, Rajdeep Singh in [7] discuss due to limited battery of sensor nodes, energy efficiency found to be a main constraint in Wireless Sensor Network. Therefore main effort has been done to enhance the network stability period and life time by minimizing the energy consumption. To achieve these objectives they proposed a technique in which, an integrated scheme will work in such a way that, selection of $\mathrm{CH}$ will be made on the basis of three level decision tree. In this, residual energy is considered one of the main parameter in decision tree; protection mechanism is also used to maintain the balance between clusters. Location of base station is also optimized in such a way that base station will be placed in high node density region at runtime. Proposed technique is deliberate and executed in MATLAB.

Advantage Proposed algorithm provides better results than the existing clustering protocols.

Future improvement is use of fuzzy based cluster head selection for cluster head selection enhancement.

Liu Huiyun in [8] has discussed LEACH algorithm as a classic clustering algorithm is widely used in wireless sensor networks, but it does not considers the number of cluster head, monitoring areas and other factors, and the network greatly huge consumption reduces the network life cycle. To these defects, on the basis of the optimal coverage theorem in Wang, one kind of CDE-LEACH algorithm is proposed, which pre-built data table to storage optimal coverage location coordinates the ideal cluster head in the base station, and to combine with the guarantee of minimum network energy consumption goal to select optimal cluster head, and to improve drawbacks of randomly selected cluster head of the LEACH algorithm. Experimental simulation platform of the Matalab7.0 is set up, the CED-of LEACH algorithm simulation is compared with LEACH algorithm results.

Advantage The network energy consumption is greatly reduced, and the network life cycle is prolonged.

Ananya Patra and Sonali Chouhan in [9] have introduced a cluster head selection process and cluster formation algorithm by re-selection of $\mathrm{CHs}$ called Improved Energy Efficient Hybrid Clustering Scheme (IEEHCS). In the proposed scheme, energy efficient $\mathrm{CHs}$ are selected by a centralized algorithm based upon remaining energy, node density and minimum separation distance to minimize the control message overheads. The key idea is that the $\mathrm{CH}$ role is repeated with the same settings or shifted to a eligible member nodes instead of re-clustering the whole network at every round. In this way, IEEHCS minimizes the frequency of updating $\mathrm{CHs}$, avoids unnecessary re-clustering in every round and saves significant amount of node energy. Results of simulation demonstrate that the IEEHCS effectively minimizes the energy consumption and prolongs the network lifetime for first node death up to $45.39 \%$ and $11.36 \%$ over LEACH-C and EEHCS, respectively, in certain network settings. 
Advantage of this proposed scheme is efficient $\mathrm{CH}$ reselection instead of re-clustering every time.

Djamila Mechta, Saad Harous, Ismahane Alem, Dounya Khebbab in [10] have proposed a new protocol which respects the energy constraints, nodes' life duration and packets reception. This work is based on the protocol LEACH-C (Low Energy Adaptive Clustering Hierarchy) which uses a centralized architecture to select cluster-heads while involving the base station and the sensors' local information. It addresses the isolation problem of remote nodes that fail to communicate their information (coordinates and residual energies) to the base station. This results in an inefficient group formation that leads to a huge loss of data and therefore the network loses its performance. For this, authors have proposed a solution that takes into account the remote nodes during the formation of groups. This new protocol, named LEACH-CKM, uses the K-means classification method to group the nodes and Minimum Transmission Energy routing protocol (MTE) for routing the information from remote nodes.

Advantage of used algorithm is that it prolongs the overall network lifetime by $30 \%$

Abedelhalim Hnini, Abdellah Ezzati, Abdelmajid Hajami [11] "Effect of a Node's Death on behaviour of the LEACH Protocol and its Descendants" This paper describes comparative analyses of LEACH protocol with its various descendants in the level on the network influence after the event of the death of a node regardless the node $(\mathrm{C}-\mathrm{H}$ node or member node). In addition, authors have given some relief proposals to strengthen the network in particular cases and its implementation in Network Simulator (NS2).

\section{PROBLEM STATEMENT}

Technological advances achieved in recent years in the field of computer networks have enabled the development of new types of low-cost smart sensors which can be configured to form autonomous networks. These sensors have particular characteristics (low storage capacity, a standalone energy source, etc.). They access the network through a wireless communication interface. They must be robust and need to be able to survive in extreme conditions due to their environment. Wireless sensor nodes fast decreasing of Energy because each node in ideal, sleep transmission and receiving mode and discharge the energy of sensor node. In this dissertation we proposed A efficient energy utilization base cluster head selection and increased life time of the sensor network, proposed work analyze with the help of network simulator-2 and identified the died node, total energy utilization, network life time, power consumption by each sensor node, throughput, packet delivery ratio and routing overhead.

\section{PROPOSED WORK}

As many researcher designed protocol in Wireless Mobile Sensor Network and minimize the energy utilization of the network in that contrast cluster base region creation is one of the major energy efficient technique. So our aim to design efficient energy utilization base cluster head selection for increasing life time of the network, for that work achievement, initially we deploy the mobile sensor node, define antenna type, routing protocol and Mac standard after that create sender and receiver node. Next phase is a cluster head selection, for that case we generate the election message that contain the energy of itself and pass to the next hop, that packet received by the new hop and compare with energy of all other node with self if their energy is higher than the other than, that node win as cluster head else maximum energy contain node in the table as cluster head. After cluster head selection, cluster head node broadcast wining message to all other sensor node, next phase is a routing phase in that case sender sensor generate the route request packet and search the path from sender to receiver and cluster head takes the decision as maximum energy contain as well as minimum power consumption sensor node as a routed nodes and select the path. After the path selection we send the data from sender to receiver and create the output files name as trace file and NAM (network animator file) with the help of trace file we retrieve the energy utilization of each sensor node, cluster head information and transmission and receiving energy use by nodes as well as node motion information from one zone to other, packet delivery ratio and throughput etc.

\section{SIMULATOR USED}

Network Simulator (Version 2), widely known as NS2 is an event driven simulation tool that is useful in studying the dynamic nature of communication networks. Simulation of wired as well as wireless network functions and protocols (e.g., routing algorithm like AODV) can be done using NS2. In general, NS2 provides users with a way of specifying such network protocols and simulating their corresponding behaviors.

\subsection{Simulation Parameters}

The NS instructions can be used to define the topology structure of the network and the motion mode of the nodes, to configure the service source and the receiver etc.

Table 1. Simulation Parameters

\begin{tabular}{|c|c|}
\hline Simulator Used & NS-2.34 \\
\hline Number of nodes & 100 \\
\hline Dimension of simulated area & $800 \mathrm{~m} \times 600 \mathrm{~m}$ \\
\hline Energy Protocol & E-LEACH \\
\hline Simulation time & 100 sec. \\
\hline Traffic type (TCP \& UDP) & CBR (3pkts/s) \\
\hline Packet size & 512 bytes \\
\hline Number of traffic connections & 5,30 \\
\hline Node movement at maximum & random (20 m/s) \\
\hline Speed & $250 \mathrm{~m}$ \\
\hline Transmission range & 1.5 Joule \\
\hline Receiving Energy & 1 Joule \\
\hline Ideal Energy & .01 Joule \\
\hline Sense Power & $\mathbf{. 1 7 5}$ Joule \\
\hline
\end{tabular}




\section{SIMULATION RESULTS}

The results analysis in case of proposed cluster based LEACH protocol and normal routing (old) detail description is mentioned and observes that the proposed is better for efficient energy consumption in WSN.

\subsection{Alive Nodes Calculation}

The routing packets in network are consumes energy it means minimum number of routing packets are deliver maximum amount of data packets in efficient routing. This graph represents the alive nodes analysis in case of proposed cluster based scheme and normal cluster based routing at simulation time of 300 seconds. In this graph we clearly notice the smooth depletion of energy from initial energy to energy remain in nodes after the end of simulation time. It means the proposed scheme based routing selection strategy are maintained the reliability in network.

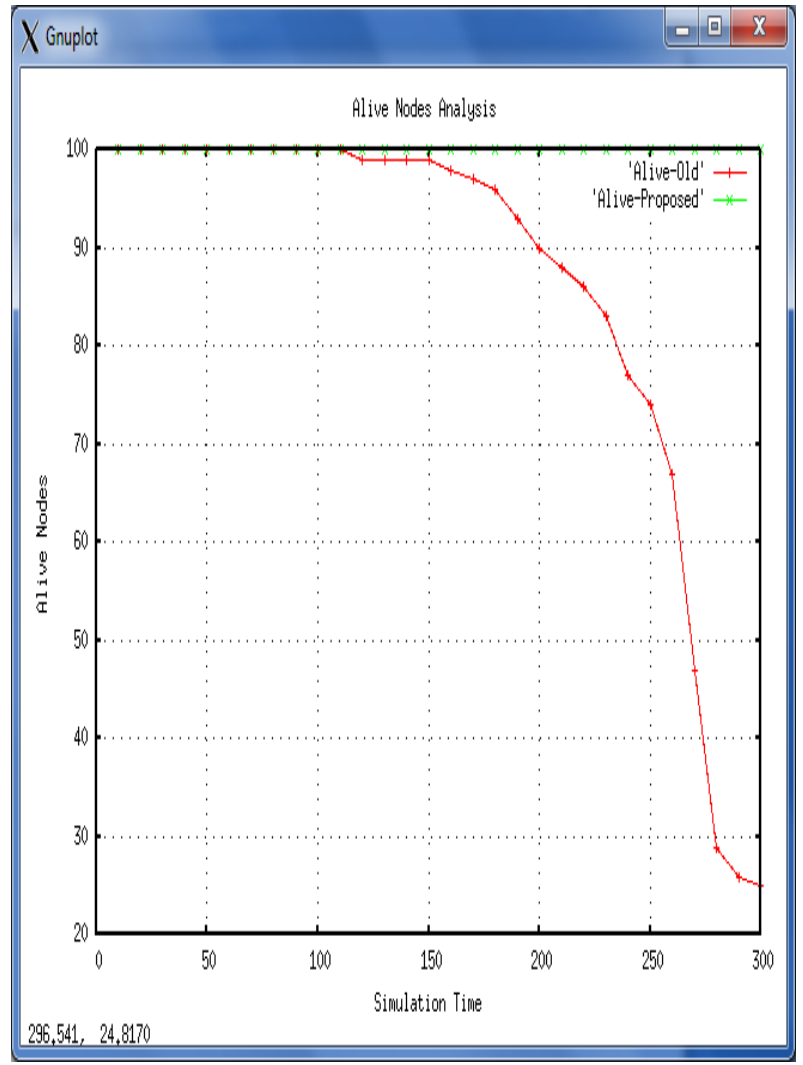

Fig 1: Number of Nodes Live in Network

\subsection{Packet Delivery Ratio Performance Analysis}

The Packet Delivery Fraction (PDR) analysis is represents the successful percentage of data received at destination. This graph is represents the packet percentage in case of proposed energy based routing and previous normal energy shortest path selection routing. Here this graph represents the more PDF in proposed LEACH cluster based routing it is about $100 \%$ but the routing load in normal cluster formation are 80 $\%$ up to end of simulation. If the routing load in network are more it means energy consumption are more by that the life of nodes are lost early as compare to proposed. It means PDF value is good not show that the overall performance of network are also better.

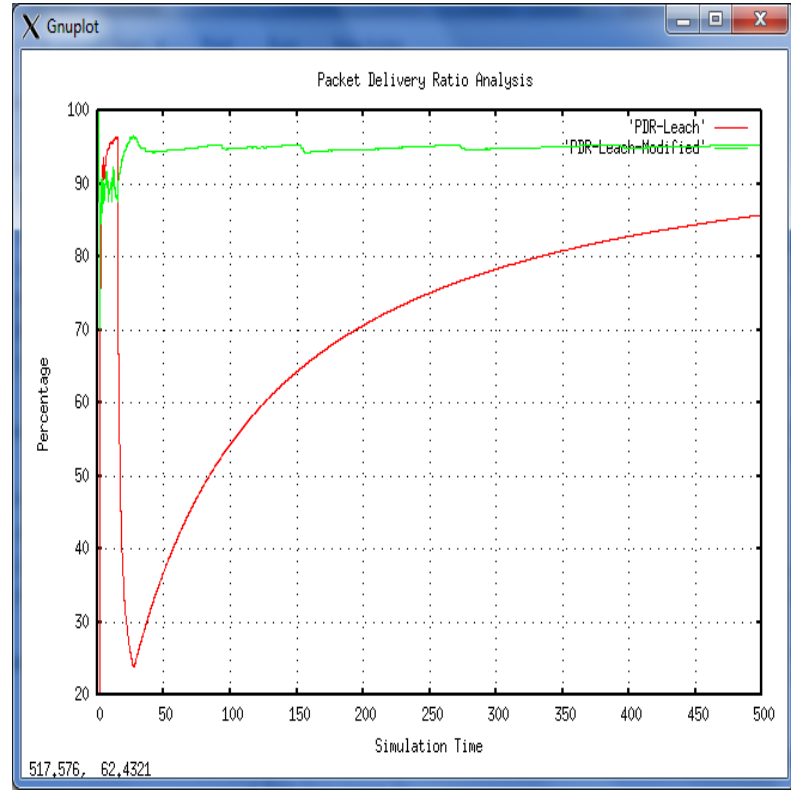

Fig 2: PDR Performance Analysis

\subsection{Number of Routing Packets Flooding Analysis}

Routing packets in network are required to established connection in between source and destination. First routing packets are established connection with destination if destination replies to sender by connection confirmation packet. The routing packets in network are consumes energy it means minimum number of routing packets are deliver maximum amount of data packets in efficient routing. In this routing packets are required then energy also required for routing packets transmission and in proposed LEACH cluster based routing with multipath the cluster formations are more that reduces consumption and minimizes the routing overhead.

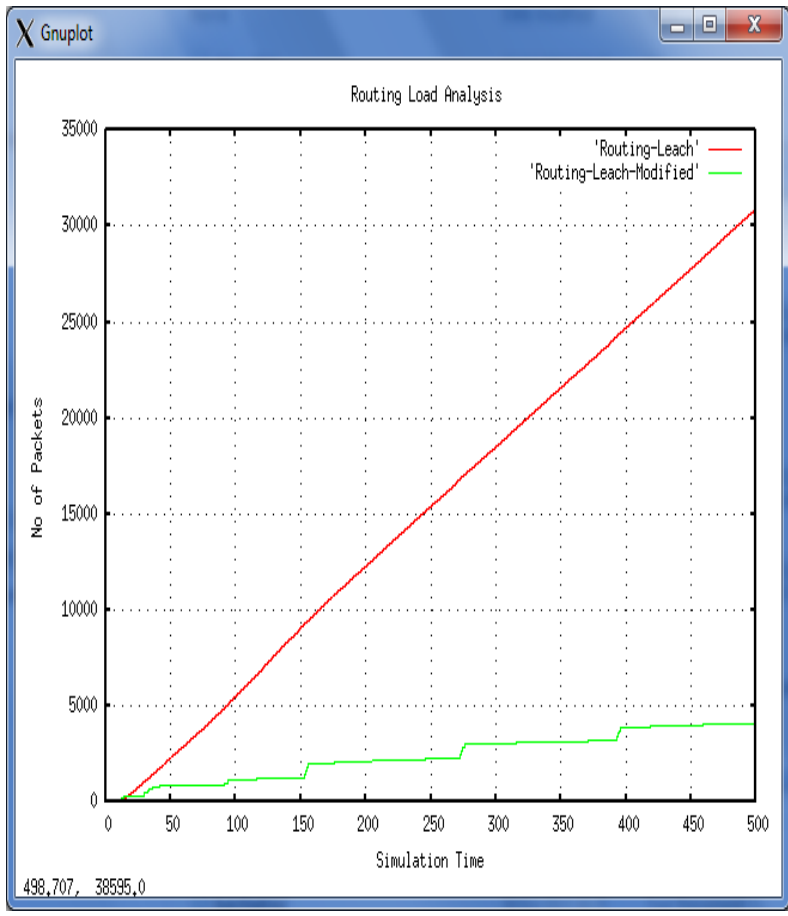

Fig 3: Packets Flooding Analysis 


\subsection{Throughput Performance Analysis}

The better throughput performance shows the better sending and receiving of data packets at sender end and receiver end. This graph represents the throughput of old and proposed LEACH with multipath routing cases. Here we clearly visualized that the throughput in case of proposed cluster based LEACH protocol are more. The throughput in case of Energy based AOMDV protocol are lower than the proposed scheme. It means that the proposed LEACH protocol is increases the energy utilization with multipath protocol. Now the results in case of normal routing is enhance the possibility of energy consumption but proposed reduces to control energy consumption.

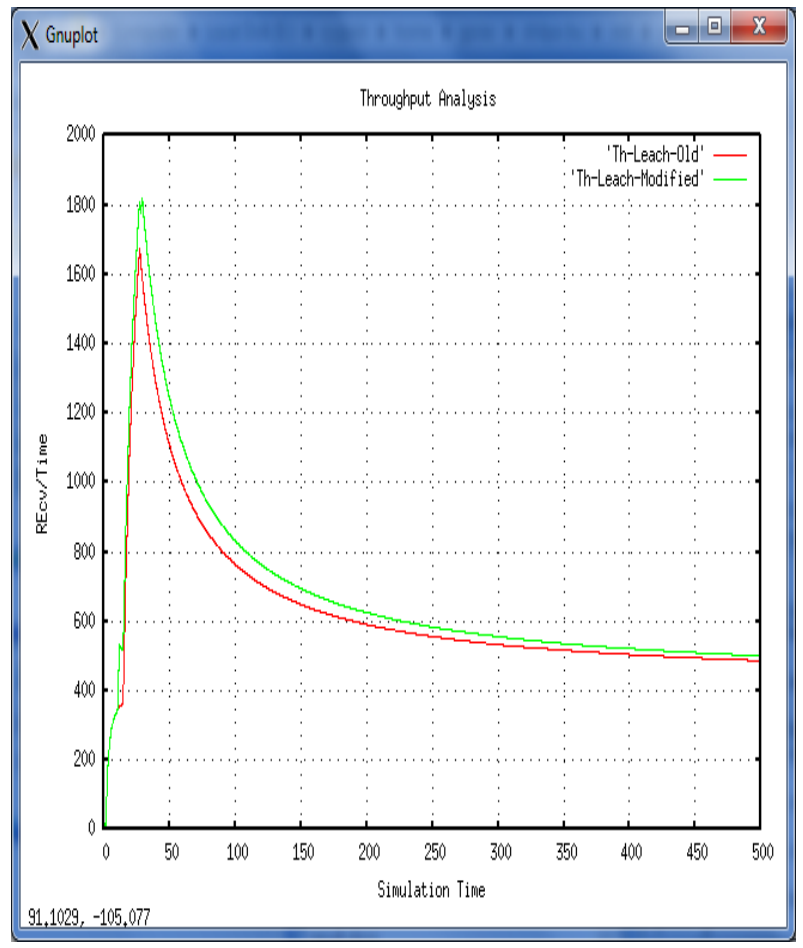

Fig 4: Throughput Performance Analysis

\subsection{Summarized Performance Analysis}

This table 2 represents the overall analysis in case of previous and proposed LEACH cluster based scheme. Here we clearly notice that in case of proposed scheme large number of packets are sending in network are more as compare to normal routing. The value of PDF is really gives appreciable performance. The reduction in routing load and delay are definitely reduces consumption of energy.

Table 2. Summarized Network Performance

\begin{tabular}{|c|c|c|}
\hline Performance & Old & Proposed \\
\hline Data Send & 14556 & 15681 \\
\hline Data Receive & 12488 & 14960 \\
\hline ROUTINGPKTS & 30911 & 4110 \\
\hline PDF & 85.79 & 95.4 \\
\hline Normal Routing Load & 2.48 & 0.27 \\
\hline No. of dropped data & 2068 & 721 \\
\hline
\end{tabular}

\section{CONCLUSION \& FUTURE WORK}

The Wireless Sensor Network is the collection of nodes or battery dependent devices that are forming temporary infrastructure for communication in decentralized manner. The all sensor devices are get-together and forming dynamic link for data delivery. The multipath routing protocol are reduces the possibility of link breakage because of nodes leave the particular link and heavy load condition. The energy or power in sensor nodes is limited and it is necessary to utilize it efficiently for better network life time. In this research we proposed a modified version of LEACH protocol in WSN. The proposed scheme is selected the reliable nodes is term of higher energy level by that the strong link is established that reduces data drop possibility due to energy deficiency. In proposed LEACH protocol the number of nodes live in network are remains more, it means the nodes are ready for further communication in WSN. The normal LEACH protocol is not provides the efficient routing by that the unnecessary energy consumption is identified that degrades the life time of network. The Cluster Head $(\mathrm{CH})$ and cluster member analysis is measured up to 500 simulation time and observe that the number of $\mathrm{CH}$ is more count in different time interval and number of members in $\mathrm{CH}$ is also less but in proposed the measurement is just apposite i.e. the sign of energy efficient routing. The rest of the performance metrics like throughput and routing overhead are showing the better routing performance in proposed LEACH as compare to normal LEACH.

In future we also modified again the concept of LEACH by including the concept of mobility factor in WSN. The mobility factor is also the major reason of link breakage but if we select the nodes that have minimum mobility for communication then the possibility of nodes out of coverage area is reduced. Another contribution is that also use the GPS system to identify the actual allocation of sensor nodes that will reduces the routing overhead cost of protocol in WSN

\section{REFERENCES}

[1] Aarti jain , B.V.R.Reddy "Sink as cluster Head: An Energy Clustering method for Wireless Sensor Networks. pp. 4799-4674-7/14/IEEE-2014.

[2] P Ipsita Panda "A Survey on Routing Protocols of MANETs by Using QoS Metrics" International Journal of Advanced Research in Computer Science and Software Engineering, Volume 2, Issue 10, pp. 121-129, 2012.

[3] C. Karlof and D. Wagner, Secure Routing in Sensor Networks: Attacks and Countermeasures, In Proc. of First IEEE International Workshop on Sensor Network Protocols and Applications, 2003.

[4] M. Tripathi,, M.S. Gaur, V. Laxmi and R.B. Battula "Energy Efficient Leach-C Protocol For Wireless Sensor Network" Malaviya National Institute of Technology, Jaipur, India pp. 402-405, 2013.

[5] Jingqing Wang and Xi Zhang "AQ-DBPSK/DS-CDMA Based Energy-Efficient and Interference-Mitigation Scheme for 3D Clustered WCSNs With Minimum Coverage Rate Constraint" 2014 IEEE Military Communications Conference.

[6] Jitender Grover, Reena Rani "Probabilistic Density Based Adaptive Clustering Scheme to Improve Network Survivability in WSN" 5th ICCCNT 2014 July 11- 13, 2014, Hefei, China. 
[7] Vinay Kehar, Rajdeep Singh "Evaluating the Performance of Reactive I-LEACH", 2014 IEEE.

[8] Liu Huiyun "An Optimized LEACH algorithm in Wireless Sensor Network" 2014 Fifth International Conference on Intelligent Systems Design and Engineering Applications 978-1-4799-4261-9/14 \$31.00 (C) 2014 IEEE.

[9] Ananya Patra and Sonali Chouhan"An Approach to Improved Energy Efficient Hybrid Clustering in Wireless Sensor Networks" 978-1-4799-4665-5/14/\$31.00 @2014 IEEE.
[10] Djamila Mechta, Saad Harous, Ismahane Alem, Dounya Khebbab "LEACH-CKM: Low Energy Adaptive Clustering Hierarchy protocol with K-means and MTE" 978-1-4799-7212-8/14/\$31.00 @2014 IEEE.

[11] Abedelhalim Hnini, Abdellah Ezzati, Abdelmajid Hajami "Effect of a Node's Death on Behavior of the LEACH Protocol and its Descendants" 2014 Fifth International Conference on Next Generation Networks and Services (NGNS) May 28-30, 2014, Casablanca, Morocco 978-14799-6937-1/14/\$31.00 @2014 IEEE

[12] The Network Simulator ।-ns-2 http://www.isi.edu/ nsnam /ns/ 